\title{
Irakische Autoren im Migrationsland Deutschland
}

\author{
Mohammad Ismail Shibib
}

Man kann mit Recht sagen, dass die gegenwärtige irakische Literatur in der Migration entstanden ist. Den Schwerpunkt dieses Überblicks bildet daher das literarische Schaffen irakischer Autoren in der deutschen Migration und deren Verhältnis zur Heimatliteratur. Dabei liegt das Hauptaugenmerk auf den Erzähltexten, besonders dem Roman, denn während die Lyrik jahrhundertelang die Literatur im arabischen Sprachraum im Allgemeinen und im Irak im Besonderen dominierte, bildet nun der Roman eine Leitgattung des gegenwärtigen Literaturbetriebs im Irak. Erst seit Beginn des 20. Jahrhunderts entwickelte sich im >Lyrikland < Irak eine neue Kunst bzw. Erzählkunst. Verschiedene Faktoren haben die Entstehung und Entwicklung dieser neuen Kunst beeinflusst.

\section{Der irakische Roman in der Migration}

Die Ursachen für die Auswanderung irakischer Autoren ${ }^{1}$ sind vielfältig: Die Flucht vor politischer Verfolgung, die Suche nach besseren Lebensbedingungen oder nach literarischer und intellektueller Freiheit sowie die strenge, willkürliche Zensur sind häufig aufgeführte Beweggründe. Die Ursachen für die Migration aus dem Irak beschränken sich somit nicht nur auf politische Gründe, sondern es spielen, vor allem nach dem Ersten Golfkrieg, auch Sicherheit und wirtschaftliche Gründe eine Rolle, da die Wirtschaftsblockade zur Verschlechterung der irakischen Wirtschaft nach dem Krieg geführt hat (vgl. Haddad 2004: 9).

Die irakische Migrationswelle begann mit der Machtergreifung durch die Baath-Partei im Jahre 1968, aufgrund der Maßnahmen gegen die Gegner der brutalen Diktatur Saddam Husseins, und nahm während des Irak-Iran-Kriegs (1980-1988) und des Zweiten Golfkriegs (1991) zu (vgl. Ibrahim 2009). Unter den Emigranten waren hervorragende Schriftsteller, die mit ihren im Ausland geschriebenen Texten auch die irakische Literaturszene geprägt haben. Lutfi Haddad schreibt in seinem Buch Anthologie der zeitgenössischen arabischen Exilliteratur: »Wenn die Migration und Migrationsliteratur zu Beginn des 20. Jahrhunderts amerikanisch und libanesisch geprägt war, ist die neue Migration europäisch und irakisch geprägt«. Und weiter heißt es: »Wenn der palästinensische

1| Mit den Bezeichnungen ,Autor،, 'Schriftsteller،, 'Dichter، ist grundsätzlich die generische Verwendung des Maskulinums verbunden. 
Schmerz älter und tiefer ist und die arabische Literatur mit zahlreichen Werken bereichert hat, so hat der irakische Schmerz die gegenwärtige Migrationsliteratur geschaffen.« (Haddad 2004: 9, 22) Frank Hessenland (2006) merkt dazu an: »Die irakische Migrationsliteratur hat in den letzten 40 Jahren maßgeblich die Entwicklung der arabischen Sprache und Dichtung bestimmt.«

Nach dem Fall der Diktatur Saddam Husseins und dem Ende der wirtschaftlichen und kulturellen Okkupation von 1990 bis 2003 konnten im Irak verbliebene Schriftsteller die Texte ihrer Kollegen im Ausland lesen. Sie hatten auch Zugang zu Weltliteratur. Sie wurden von der Angst vor dem Diktator befreit und begannen selbst, über verschiedene Themen zu schreiben. Es fällt auf, dass eine große Anzahl irakischer Romane sowohl im Irak als auch in der Emigration nach 2003 geschrieben und publiziert worden ist. Bemerkenswert ist ferner, dass sich der irakische Roman nach 2003 vor allem auf die Erzähltechnik konzentriert. Dabei experimentieren die Autoren mit neuen Erzähltechniken auf den Ebenen der Form sowie der Sprache und entwickeln auf diese Weise neue Visionen, Gedanken und Themen.

Die in den Migrationsländern erschienenen Romane wie Irakische schwarze Monde in Schweden (2003) von Ali Abdulaal und Unter dem Himmel Kopenhagens (2010) von Hawraa Al-Nidawi haben das Leben in der Migration und die damit verbundenen psychischen und sozialen Auswirkungen zum Thema. Das Problem der Identität wird in Warid Badir Al-Salems Werk Die Wunder von Bagdad (2012) thematisiert. Interkulturalität, Probleme der Minderheiten, soziale Probleme, die politische Realität und die Verfälschung der irakisch-arabischen Geschichte, die heute noch eine umstrittene Geschichte ist (vgl. Thiab 2014), sind die Themen in den Romanen Der Krieg im Vergnügungsviertel (1993) von Najem Wali, Die Nacht des Landes (2002) von Jassim Hillawi und nicht zuletzt Der Letzte der Engel (2014) von Fadhil Al-Azzawi.

Die meisten in der Migration geschriebenen irakischen Romane setzen sich indes mit den Alltagsszenen und den Alltagsgeschichten im Irak auseinander, z.B. Der Tabakwächter (2008) von Ali Badir, Marines Milch (2008) von Awad Ali, Die Amerikanische Enkelin (2009) von Inaam Kachachi, Die Toten Bagdads (2008) von Jamal Hussein Ali und Ein Iraker in Paris (2012) von Samuel Shimon.

Der Tabakwächter (2008) von Ali Bader ist ein hervorragendes Beispiel für die sich wandelnden Erzähltechniken des Migrationsautors. Herkunftsland und Migrationsland gehen hier ineinander über. Die Hauptfigur, ein Jude, ist nicht in der Lage, sein Heimatland, den Irak, zu verlassen, geschweige denn zu vergessen. Er kann auch nicht in einem anderen Land, z.B. in Israel, leben. Doch selbst im Irak kann er nur dann leben, wenn er einen anderen Namen, eine andere Religion bzw. einen anderen Glauben annimmt und somit seine Identität verleugnet. Dadurch erlebt er eine Art innere Verbannung, die über die geographische Semantik der Migration hinausgeht.

Die Amerikanische Enkelin (2009) von Inaam Kachachi thematisiert die Identitätsproblematik der ethnischen und religiösen Minderheiten im Irak. Die Zugehörigkeit dieser Minderheiten wird erschüttert, wenn Chaos herrscht inmitten der Hauptgruppen, die diese Minderheiten umfassen. Die engen Identitäten 
werden gelöscht oder stattdessen breite kosmopolitische Identitäten vorgeschlagen.

Der Krieg und seine Folgen für den Einzelnen sowie die ganze Gesellschaft ist ein wichtiges Thema des irakischen Romans. Beispiele dafür sind Najem Walis Roman Der Krieg im Vergnügungsviertel (1993) und Die Welt minus eins von Maysaloon Hadi (vgl. Ash-Shibibi 2014).

In Hadis Roman geht es um das Leben im Schatten des Golfkriegs und seine Folgen. Der Roman befasst sich mit der Wirkung des Kriegs auf die irakische Bevölkerung, auf den Körper und den Geist des Einzelnen sowie mit den Tragödien des Schmerzes und dem Gefühl des Verlustes.

Wali hingegen thematisiert die Emotionen der Rekruten, ihre Flucht und ihre Suche nach besseren Lebensalternativen. Er beschreibt ein Leben, das von der Gleichheit des Vergnügungsviertels, der Welt des Gesangs und des Sexes sowie dem Krieg mit all seinen Grausamkeiten bestimmt wird.

Ein anderes wichtiges Thema, das die meisten irakischen Romanautoren aufgegriffen haben, ist die Besatzung und die blinde Gewalt. Die Autoren stellen die Brutalitäten der Kriege dar, die der Irak erlebt hat, und wie diese Kriege die moralischen Werte im Irak aufgelöst und im Land zu kriminellen Akten, wie Entführungen und Geiselnahmen, geführt haben. Das zeigt u.a. der Roman Die drei Freunde (2006) von Shakir Khassbak. Khassbak setzt sich darin mit diesem neuen Phänomen in der irakischen Gesellschaft auseinander. Die Entführung ist der Verknüpfungspunkt der Schicksale von drei Freunden, die drei verschiedenen Religionen angehören: Ali ist Muslim, Joseph ist Christ und Zaki ist Sabit.

Auch Einzelschicksale haben sowohl die im Irak als auch in der Migration lebenden irakischen Schriftsteller fasziniert. Jeder Mensch wird allein mit seinem Schicksal in einer Welt konfrontiert, die den Bezug zu Werten und Gerechtigkeit völlig verloren hat, wie in Ahmed Khalafs Roman Fancy Halter (2005), den man als psychologischen Roman bezeichnen kann. Auch andere Romanschriftsteller haben die psychische Realität ihrer Figuren aus psychologischer Sicht behandelt, wie Asa'ad Al-Lamis in Eminas Fenster, in dem er die Psyche einer terroristischen Persönlichkeit darstellt.

Der irakische Roman hat, wie die Werke u.a. von Najem Wali und Hussein Al-Mozany zeigen, die drei Tabus Sex, Politik und Religion überwunden und versucht, das Unsagbare zu sagen. Bis 2003 war es schwierig, im Irak über Sexualität, Politik und Religion frei und offen zu schreiben. Eine Ausnahme in diesem Zusammenhang bilden die Romane der in der Migration lebenden irakischen Autoren.

\section{Zwischenbilanz}

Im Allgemeinen kann man folgende Tendenzen in der zeitgenössischen irakischen Literatur in der Migration, besonders in der Erzählprosa, unterscheiden:

1. Die Schriftsteller konzentrieren sich auf ihre persönlichen Geschichten, Familien und Gesellschaften. Sie drücken sich in ihren Arbeiten zum ersten 
Mal frei und individuell aus (vgl. Hessenland 2006). In diesem Zusammenhang kann auf den Roman Auf der Bananenmatratze (2006) des 2013 verstorbenen Abdulsattar Nassir (1947-2013) hingewiesen werden. Die vom Erzähler aufgeschriebenen Erinnerungen bilden den Hauptteil des Romans.

2. Die meisten Autoren haben sich von einer bildreichen poetischen Sprache distanziert und damit begonnen, eine einfache direkte Sprache und kurze Sätze zu verwenden.

3. In ihren Texten haben die Autoren die Realität realistisch-fiktional beschrieben. Damit haben sie versucht, Alternativen zur vorgestellten Welt darzustellen.

4. Der gegenwärtige irakische Roman ist gekennzeichnet durch die Abfolge von Ereignissen, was als Alternative zum statischen irakischen Roman vor $2003 \mathrm{zu}$ sehen ist, sowie durch das Überlappen von Vergangenheit und Gegenwart, Realität und Fiktion.

\section{Irakische Autoren in deutscher Migration}

Die in der deutschen Migration lebenden irakischen Autoren kann man in vier Gruppen einteilen:

1. Autoren, die ihre Texte nur auf Arabisch geschrieben haben, wie Salima Salih, Sabri Hashim und Fadhil Al-Azzawi,

2. Autoren, die ihre Texte auf Arabisch verfasst haben und sie ins Deutsche übersetzen lassen, wie Najem Wali und Khalid Al-Maaly,

3. Autoren, die nur auf Deutsch geschrieben haben, wie Abbas Khider und Hikmat al-Sabty,

4. Autoren, die zuerst in arabischer Sprache geschrieben und daneben moderne deutsche Autoren aus dem Deutschen ins Arabische übersetzt haben und im Laufe ihres Aufenthalts in Deutschland dazu übergegangen sind, auch die eigenen Werke in deutscher Sprache zu verfassen, wie Hussein Al-Mozany.

Während einige Autoren, wie Sabri Hashim, Salima Salih, Fadhil Al-Azzawi, Jamil Hussein Al-Saadi oder Abbas Khider, von Anfang an sowohl Gedichte als auch narrative Texte verfasst haben, gingen andere dazu über, nur Romane und Novellen zu schreiben, wie Hussein Al-Mozany und Najem Wali. Eine Ausnahme bildet in diesem Zusammenhang Khalid Al-Maaly. Er schreibt immer noch hauptsächlich Gedichte und übersetzt auch die Gedichte arabischer Lyriker ins Deutsche. Außerdem ist zu erwähnen, dass die meisten Romane von Sabri Hashim und Jamil Al-Saadi in >lyrischer Sprache< geschrieben worden sind.

Da sich die Studien zur Migrationsliteratur in Deutschland hauptsächlich mit den in der Migration geschriebenen Romanen befassen und die meisten in der deutschen Migration lebenden irakischen Autoren von der Lyrik zum Roman übergegangen sind, da sie die Meinung vertreten, mit dem Roman größe- 
re Freiheit $\mathrm{zu}$ haben, konzentriert sich auch die folgende Darstellung der Texte der in der deutschen Migration lebenden irakischen Autoren auf den Roman.

Die in Deutschland lebenden irakischen Autoren unterscheiden sich bezüglich der von ihnen aufgegriffenen Themen und Erzählweisen nicht sehr von den im Irak und in der europäischen Migration lebenden Autoren, und anhand ihrer Werke lassen sich keine klaren Tendenzen ausmachen. Im Allgemeinen kann man jedoch einige Gemeinsamkeiten in ihren Texten feststellen.

Die meisten Texte sind autobiographisch und setzen sich mit dem Leben im Irak während der Regierungszeit Saddam Husseins und nach dem Sturz seines Regimes auseinander, wie Abbas Khiders Gefängnisroman Der falsche Inder (2008) und das Frühwerk von Fadhil Al-Azzawi Die fünfte Burg Al-Qal'a al-khamisa (1972), der als erster irakischer Gefängnisroman gilt.

In dem Falschen Inder ${ }^{2}$ werden in einer tragisch-komischen Weise die Haft und Folter während der Diktatur Saddam Husseins, die Flucht eines jungen Irakers und die damit verbundenen Erlebnisse zum Thema. Der Erzähler findet im Zug von Berlin nach München ein arabisches Manuskript mit dem Titel Memorien, das die Fluchtgeschichte eines jungen Irakers namens Rasul Hamid erzählt. Auf seinem Weg trifft er Flüchtlinge aus aller Welt, die wie er auf der Suche nach einem Leben ohne Hunger und Krieg sind und dafür sehr viel opfern.

Flucht ist auch ein Thema in Hussain Al-Mozanys Texten. Im Jahre 1989 hat er erste Gedichte in deutscher Sprache in der von Anja Tuckermann herausgegebenen Anthologie In die Flucht geschlagen. Geschichte aus dem bundesdeutschen Asyl veröffentlicht (vgl. Tuckermann 1989). In arabischer Sprache verfasste er wiederum unter dem Titel Der Herbst der Städte (1996) Kurzgeschichten sowie den Roman Das Geständnis des Fleischhauers (2007). Seine Romane Der Marschländer: Bagdad, Beirut, Berlin (1999) und Mansur oder Der Duft des Abendlandes (2002) sind die literarische Verarbeitung seiner Flucht aus dem Irak und der Emigration in die Fremde.

Die politische und gesellschaftliche Lage im Irak ist in den Werken irakischer Autoren der Migration immer präsent. Abbas Khiders zweites Werk Die Orangen des Präsidenten (2011) setzt sich mit den großen Auswirkungen des Embargos auseinander, das die Bevölkerung Iraks von 1990 bis 2003 erlebt hat. Najem Walis Roman Ein Ort namens Kumait - die Geschichte einer Beschneidung (1997) behandelt einen Tabubruch, indem darin die Beschneidung der Männer als Folter bezeichnet wird. Der Tabubruch wiederholt sich in Walis im Jahre 2009 er-

2 | Die Titel, die Abbas für seine Romane auswählt, sind nicht einfach zu deuten, wenn ihr Kontext unbekannt ist. Der Falsche Inder spielt auf die Bezeichnung an, die das Regime Saddam Husseins für die irakische Bevölkerung, besonders im Süden, als Schimpfwort verwendet hat. Gemeint ist, dass die Einwohner im Süden Iraks keine echten Araber sind, sondern ihre Vorahnen Inder, die mit den britischen Truppen während der Besatzung des Iraks durch die Briten ins Land gekommen waren. Ein Brief in die Auberginenrepublik ist eine Anspielung auf die Blockadezeit, in der die meisten irakischen Familien fast jeden Tag nur Auberginen gebraten oder gekocht haben, sodass sich im Irak eine Ironiebezeichnung verbreitete, und zwar ,Pfannenmonster. 
schienenem Roman Reise in das Herz des Feindes: Ein Iraker in Israel (deutsche Übersetzung aus dem Arabischen von Imke Ahlf-Wien). Mit seinem Roman Engel des Südens (2011 ins Deutsche übersetzt von Imke Ahlf-Wien) führt uns der Schriftsteller in die jüngste Geschichte des Iraks zurück und schildert das Leben in der südlichen Amaria (bzw. Amarah) im Irak, wo die Ethnien und Religionen vor 2003 friedlich zusammenlebten. Durch die Enthüllung der Lebensund Liebesgeschichten seiner Figuren zeigt uns Najem Wali in seinem Roman Die Reise nach Tell al-Lahm (2004) ein von der Diktatur und zwei Kriegen gezeichnetes Land.

Sabri Hashim schreibt in einer für die arabische bzw. irakische Literatur neuen Erzähltechnik, indem er mythologische wie geschichtliche Elemente und Symbole verwendet, um die Ereignisse im Irak in den vergangenen Jahrzehnten darzustellen und aus philosophischer Sicht zu beleuchten. Das zeigt sich in allen seinen Texten, wie Die Mulatten (2000), Trüffelausspruch (2005) und Huraki, das Land der Assyrer (2008), besonders aber in der Gitarre von Median (2009).

Die Migrationsromane sind in Wirklichkeit Romane über das Heimatland. Dieser verlorene Ort der Heimat bildet das Schreibmotiv, nicht der aktuelle, jetzige Ort. Die These wird unterstützt durch die Titel und Handlungsorte der Texte der in der deutschen Migration lebenden irakischen Autoren. Najem Walis Romane zeigen das: z.B. Der Krieg im Vergnügungsviertel (1989), Die Reise nach Tell al-Lahm (2004), Ein Ort namens Kumait (1997) und sein Roman Bagdad Marlboro (2014), aus dem Arabischen übersetzt von Hartmut Fähnrich. Was erzählt wird, ist die Begegnung des Erzählers mit einem Unbekannten in Bagdad, wo ein ehemaliger Leutnant der US-Armee während des Zweiten Golfkriegs an der Tötung wehrloser irakischer Soldaten teilnimmt. Nun möchte der Ex-Offizier dem Erzähler ein Heft übergeben, in dem die Träume und Wünsche der getöteten Soldaten verzeichnet sind. Damit will er Buße tun und die Opfernamen vor dem Vergessen retten.

Die Figuren des Romans Bagdad Marlboro standen mindestens vier Kriege innerhalb von fünfundzwanzig Elendsjahren durch: Der Kurdenkrieg (von 1961 bis 1970), der Irak-Iran-Krieg oder der Erste Golfkrieg (von 1980 bis 1988), der Kuwait-Krieg oder der Zweite Golfkrieg (August 1990 bis Februar 1991) und der Dritte Golfkrieg (von März bis Mai 2003), der anders als die anderen Kriege war: »Er war die Summe aller Kriege, die wir bisher erlebt haben.« (Zit. n. Löffler 2014) Diese Kriege und der irakische Bürgerkrieg (2014-2017) haben einen großen Teil des Landes zerrüttet, Werte zerstört und die Menschen im Irak traumatisiert. Najem Wali thematisiert in seinem Roman Bagdad Marlboro diese Verheerungen.

Najem Wali stellt in seinen Romanen verdrängte Erinnerungen an schreckliche Gewalterfahrungen dar, Kriege, die die Iraker traumatisiert, das Land zerrüttet und Werte vernichtet haben. Der einzige Wunsch der Romanhelden ist das Überleben im Chaos und im Angesicht des draußen tobenden Infernos von Autobomben, Selbstmordattentaten, Entführungen und willkürlichen Milizmorden. 
Wie viele von Hussein Al-Mozanys Erzählungen ist auch der Roman Mansur oder Der Duft des Abendlandes (2002) zwischen Deutschland und der arabischen Welt angesiedelt. Al-Mozany erzählt darin die Geschichte eines irakischen Soldaten namens Mansur, der versucht, die deutsche Staatsbürgerschaft zu erlangen, indem er behauptet, von einem mittelalterlichen Wandermönch abzustammen. Der Roman Das Geständnis des Fleischhauers (2007), dessen Handlungsorte Köln und Kairo sind, kann als psychologischer Roman gelesen werden.

Auch Sabri Hashim siedelt seine Romane zwischen verschiedenen Orten (Basra und Berlin) an. Das zeigt sich besonders in seinem Roman Die Mulatten (2000). Das Werk ist ein lyrischer Roman, der auf mehreren Schlüsselthemen basiert - darunter die wichtigsten Traditionen in einigen Ländern des Ostens, einschließlich die Bedeutung von >Ehre < und wie man damit umgeht. Basra und Berlin bilden eine wichtige erzählerische Verbindung: Jaber, »der Bezauberte«, verlässt seine Geliebte Siham al-Khulasiyah in Basra und flieht nach Berlin, da die Bewohner der Stadt Basra das Verhältnis missbilligen und gegen den Moralbruch eines nächtlichen Treffens der Liebenden rebellieren.

Fadhil Al-Azzawi macht den Irak der 1950er Jahre zum Thema seines Romans Der Letzte der Engel (2014) und beleuchtet allgegenwärtige Gewalt, ideologische Machtansprüche, aber auch die kulturelle Vielfalt des Landes.

\section{Fazit}

Der irakische Roman vor 2003 kann als realistischer Roman bezeichnet werden und ist nach 2003 durch ein Wechselspiel von Fiktionalität und Faktizität gekennzeichnet. Krimis und Science-Fiction sind kaum im irakischen Roman vertreten; dem arabischen bzw. irakischen Bewusstsein sind diese Genres fremd. Diese Erkenntnis erstaunt umso mehr, wenn man bedenkt, dass schon Elemente aus dem Werk Tausendundeine Nacht der Science-Fiction-Literatur zugeordnet werden könnten.

Der moderne irakische Roman hat sich erst in der Migration entwickelt. Alle emigrierten Autoren haben das Gefühl, dass sie und ihre Werke in einer doppelten Migration leben. Sie leben in einem Land, in dem sie keine oder nur eine geringe Leserschaft finden können, und so veröffentlichen sie ihre Werke in anderen Ländern, wie dem Libanon. Der Grund für diese doppelte Migration liegt möglicherweise darin, dass die Romane der meisten Autoren sich nicht mit dem Alltag und dem Leben in Deutschland auseinandersetzen, sondern mit dem Leben und dem Alltag im Irak. Sie thematisieren die Geschichten und die Geschehnisse, die die Verfasser erlebt haben und die noch in ihren Erinnerungen präsent sind. Ihre Erfahrungen sind der Erzählstoff ihrer Werke. Das Identitätsproblem in der zeitgenössischen irakischen Erzählkunst zeigt sich insbesondere in den irakischen Romanen nach der stürmischen Veränderung im Jahre 2003, welche die soziale und politische Struktur im Irak stark betroffen hat. Ein Grund für den Erfolg des modernen irakischen Romans liegt darin, dass er in der Lage ist, die drei Tabus Sex, Politik und Religion zu überwinden. 
In der irakischen Literaturszene wird derzeit über die Bezeichnung des irakischen Romans nach 2003 debattiert. Dafür werden verschiedene Bezeichnungen vorgeschlagen: > Roman nach der Wende<, > Roman nach $2003<$ und >Roman nach der stürmischen Änderung . Diskutiert wird auch, ob und wie der irakische Roman nach der Wende die amerikanische Besatzung und ihre negativen Folgen thematisiert.

Die nach 2003 erschienenen Werke haben das Ansehen der irakischen Erzählszene verändert. Mittlerweile handelt es sich um eine im arabischen Raum und in der ganzen Welt viel gelesene Literatur, besonders, nachdem die Werke von Ali Badr, Muhsin Al-Ramli, Abdulhadi Sadun, Sinan Antuan, Luay Hamza Abbas und vielen anderen in verschiedene Weltsprachen übersetzt worden sind und Autoren wie Sinan Antuan für seinen Roman Oh Mariam, Ahmed Al-Saadawi für Frankenstein in Bagdad oder Inaam Kachachi für ihren Roman Die amerikanische Enkelin den Internationalen Preis für den arabischen Roman erhalten haben (vgl. Thiab 2014).

Da die Migrationshintergründe unterschiedlich sind und von den politischen, ökonomischen sowie gesellschaftlichen Situationen in den jeweiligen arabischen Ländern abhängen, ist auch das literarische Schaffen der arabischen Migranten bezüglich des Inhalts und der Form sehr unterschiedlich. Einen Sonderfall bilden die Texte irakischer Autoren in der Migration, da sie erst in der Migration die Möglichkeit gehabt haben, frei und ohne Angst zu schreiben. Dabei stehen diese im Gegensatz zu den noch im Irak lebenden Autoren, die aus Angst vor der Diktatur und den gesellschaftlichen Normen nicht ohne Hemmungen schreiben.

\section{KuRze BIOGRAPHIE UNd BIBLIOGRAPHIE DER In DeUtSchland Lebenden IRAKISChen Autoren}

Die folgende Zusammenstellung versteht sich als erste, vorläufige Übersicht über die zentralen Lebensstationen und die literarische Produktion zeitgenössischer irakischer Exilautoren.

Fadhil Al-Azzawi, 1940 in Kirkuk geboren, hat in Bagdad Englische Literatur studiert. In den $1960 e r$ Jahren des vergangenen Jahrhunderts war er Mitbegründer einer Gruppe für avantgardistische Lyrik. Diese Gruppe setzte sich zum Ziel, die irakische Lyrik im Sinne der Moderne zu erneuern. Sie forderte auch die schriftstellerische Unabhängigkeit von jeglichem politischen Einfluss und lehnte die Versuche der Ideologisierung der Literatur ab. Nachdem Al-Azzawi den Irak im Jahre 1977 verlassen hatte, lebte er in der DDR. Dort promovierte er in Journalistik. Sein literarisches Schaffen umfasst Gedichtsammlungen, Romane, Kurzgeschichten, literaturwissenschaftliche Abhandlungen und Übersetzungen aus dem Englischen und Deutschen ins Arabische, wie u.a. Gedichte von Christian Morgenstern, Texte von Hans Magnus Enzensberger und Tilman Spengler sowie Robert Musils Roman Der Mann ohne Eigenschaften. 
Werke:

- (1998): Auf einem magischen Fest. Gedichte. Aus dem Arabischen. Berlin.

- (2000): Die fünfte Burg [1969]. Roman. Beirut.

- (2001): Die Vorfahren. Roman. Freiberg.

- (2002): Seltsame Kreaturen Fadhil al-Azzawis [1969]. Roman. Beirut.

- (2014): Der Letzte der Engel. Roman. Aus dem Arab. v. Larissa Bender. Zürich.

Während Al-Azzawi im arabischen Sprachraum seit seinen literarischen Anfängen zu den meist gefeierten und wichtigsten Autoren seiner Generation gehört, ist er in Deutschland fast unbekannt. Seine Gedichtsammlung Miracle Maker (2003) umfasst Gedichte aus sechs früheren Lyrikbänden und gibt einen Überblick über seine dichterische, humorvolle sowie melancholische Beschäftigung mit Mythologien. Im englischen Sprachraum, besonders in den USA, ist er mit der Neuauflage seines Werks Akhir al-mala'ika (1992; engl. The Last of the Angels, 2007) bekannt. Sein Roman Madina min Ramad (2011; »Stadt der Asche«) erzählt eine Liebesgeschichte vor dem Hintergrund des Sechstagekriegs. ${ }^{3}$

Khalid Al-Maaly, 1956 in As Samawa geboren, verließ 1979 aus politischen Gründen den Irak. Er lebt seit 1980 als Schriftsteller, Übersetzer und Verleger in Köln. Al-Maaly schreibt hauptsächlich Gedichte. 1990 erhielt er den Förderpreis des Landes Nordrhein-Westfalen für junge Künstlerinnen und Künstler.

Werke:

- (1989): Gedanken über das Lauwarme. Prosa. Frankfurt a.M.

- (1992): Klage eines Kehlkopfes. Gedichte. Köln.

- (1994): Phantasie aus Schilf. Gedichte. Berlin.

- (1997): Landung auf dem Festland. Gedichte. Berlin.

- Zusammen mit Mona Naggar (2004): Lexikon arabischer Autoren des 19. und 20. Jahrhunderts. Heidelberg.

- (2004): Die arabische Welt - zwischen Tradition und Moderne. Heidelberg.

- (2007): Rückkehr aus dem Krieg. Eine Anthologie zeitgenössischer Lyrik aus dem Irak. Arabisch - deutsch. Köln/Frankfurt a.M.

- (Hg.; 2008): Die Flügel meines schweren Herzens. Lyrik arabischer Dichterinnen vom 5. Jahrhundert bis heute. Aus dem Arab. übers. zusammen mit Heribert Becker. Zürich.

3 | Im Sechstagekrieg kämpften Israel und die arabischen Staaten Ägypten, Jordanien und Syrien gegeneinander. Er dauerte vom 5. bis zum 10. Juni 1967. 
Übersetzungen aus dem Arabischen ins Deutsche:

- Badr Shakir as-Sayyad (1995): Die Regenhymne und andere Gedichte. Arabisch - deutsch. Übers. zusammen mit Stefan Weidner. Berlin 1995.

- Mahmoud Darwish (1996): Weniger Rosen. Gedichte. Arabisch - deutsch. Übers. zusammen mit Heribert Becker. Berlin.

- Sargon Boulus (1997): Zeugen am Ufer. Gedichte. Arabisch - deutsch. Übers. zusammen mit Stefan Weidner. Berlin.

- Ounsi el-Hajj (1998): Die Liebe und der Wolf, die Liebe und die Anderen. Gedichte. Übers. zusammen mit Heribert Becker. Berlin.

- Abd al-Wahhab al-Bayati (2003): Aischas Garten. Ausgewählte Gedichte. Aarabisch - deutsch. Berlin.

- Saadi Yousef (2004): Fern vom Ersten Himmel. Ausgewählte Gedichte. Arabisch - deutsch. Übers. zusammen mit Heribert Becker. Berlin 2004.

- Saif ar-Rahbi (2007): Das Heulen der Wölfe. Ausgewählte Gedichte. Berlin.

Hussain Al-Mozany, 1954 in Amarah/Südirak geboren, wuchs in Bagdad auf. 1978 ging Al-Mozany in den Libanon, wo er als Journalist arbeitete. Seit 1980 lebt er in Deutschland. Er studierte in Münster Arabistik, Islamwissenschaft, Germanistik und Publizistik. Von 1991 bis 1993 hielt er sich in Kairo auf. Er veröffentlichte zahlreiche Erzählungen und Romane in arabischer Sprache und übersetzte Bachmann, Benn, Grass u.a. ins Arabische.

\section{Werke:}

- (1999): Der Marschländer. Bagdad, Beirut, Berlin. Roman. Frankfurt a.M.

- (2002): Mansur oder der Duft des Abendlandes. Roman. Leipzig.

- (2007): Das Geständnis des Fleischhauers. Roman. Berlin.

- (2011): Parallelwelten. Essay. Dresden.

Sabri Hashim, $195^{2}$ in Basra geboren, lebt seit 1990 in Deutschland und seit 1996 in Berlin. Er schreibt Romane und Gedichte.

\section{Werke:}

- (1995): Der Tanz der Skulpturen. Roman. Damaskus.

- (1995): Die Nacht des Liedes des Sängers. Erzählungen. Damaskus.

- (1997): Die Bucht des Elefanten. Roman. Damaskus.

- (2000): Al-Khalasion (Die Mulatten). Roman. Beirut.

- (2002): Das Spektrum der Taupoesie. Gedichte. Damaskus.

- (2002): Island des Wiedehopfs. Gedichte. Damaskus.

- (2005): Horky Land von Assyrien. Roman. Damaskus.

Abbas Khider, 1973 in Bagdad geboren, lebt seit 2000 in Deutschland (Berlin). Er erhielt verschiedene Auszeichnungen, 2013 wurde er mit dem Nelly-Sachs-Preis 
sowie dem Hilde-Domin-Preis für Literatur im Exil geehrt, 2017 erhielt er den Adelbert-von-Chamisso-Preis für sein bisheriges literarisches Gesamtwerk.

Werke:

- (2008): Der falsche Inder. Roman. Hamburg.

- (2011): Die Orangen des Präsidenten. Roman. Hamburg.

- (2013): Brief in die Auberginenrepublik. Roman. Hamburg.

- (2016): Ohrfeige. Roman. München.

Salima Salih, 1942 in Mosul-Irak geboren, studierte Rechtswissenschaft in Bagdad und Journalistik in Leipzig. Von 1967 bis 1969 besuchte sie das Institut für Schöne Künste in Bagdad. 1986 promovierte Salih über die internationalen Entwicklungstendenzen der Massenmedien. Seit 1978 lebt sie in Deutschland und seit 1983 in Berlin. Zwischen 1961 und 1977 arbeitete sie als Journalistin für verschiedene irakische Zeitungen und Zeitschriften. Einige ihrer Texte sind ins Englische, Persische, Spanische und auch Malayalami übersetzt. Sie ist freie Schriftstellerin und Übersetzerin.

Werke:

- (1961): Weil du ein Mensch bist (Li anka Insan). Erzählungen. Bagdad.

- (1963): Im Laufe des Lebens (Fi Rakb Al-Hayat). Erzählungen. Bagdad.

- (1974): Das Aufstehen (Al-Nuhuth). Novelle. Damaskus.

- (1975): Verwandlungen (Al-Tahawulat). Erzählungen. Damaskus.

- (1994): Blume der Propheten (Zahrat Al-Anbia). Erinnerungen. Damaskus.

- (1995): Baum der Vergebung (Schagarat Al-Maghfira). Erzählungen. Damaskus.

- (2005): Vergessen (Nisian). Gedichte. CD.

- (2006): Die Suche nach dem Khalandaq (Al-Bahth an Al-Khalandaq). Erzählungen. Beirut.

- (2013): Der Abgrund (Al-Hawiya). Novelle. Beirut.

- (2014): Kalkang oder Die tausendundzweite Nacht und die Nächte danach. Beirut.

Übersetzungen aus dem Deutschen ins Arabische:

- Ingeborg Bachmann (1995): Das dreißigste Jahr. Beirut.

- Christa Wolf (1996): Kassandra. Beirut.

- Angela Grünert (1999): Der längste Weg heißt Frieden. Beirut.

- Christa Wolf (2001): Medea. Stimmen. Beirut.

- Christa Wichterich (2002): Die globalisierte Frau. Beirut.

- Gotthold Ephraim Lessing (2002): Philotas. Berlin.

- Emilie Reute (2002): Memoiren einer arabischen Prinzessin. Beirut.

- Uwe Timm (2003): Am Beispiel meines Bruders (Auszug). Litrix.de. 
- Markus Werner (2007): Am Hang. Beirut.

- Josef von Ess (2008): Theologie und Gesellschaft im 2. und 3. Jahrhundert. Bd 1. Beirut.

Najem Wali, geboren 1956 in Basra/Irak, hat in Bagdad Deutsche Literatur studiert. 1980 flüchtete er nach Ausbruch des Iran-Irak-Kriegs nach Deutschland. Heute lebt Wali als freier Schriftsteller und Journalist in Berlin. Er ist Kulturkorrespondent der bedeutendsten arabischen Tageszeitung Al-Hayat und schreibt regelmäßig u.a. für die Süddeutsche Zeitung, die Neue Zürcher Zeitung und Die Zeit. Für seinen Roman Bagdad Marlboro wurde er 2014 mit dem Bruno-KreiskyPreis ausgezeichnet.

Werke:

- (1989): Der Krieg im Vergnügungsviertel. Roman. Aus dem Arab. v. Jürgen Paul. Hamburg.

- (1990): Hier in dieser fernen Stadt. Roman. Aus dem Arab. v. Thomas Schade. Hamburg.

- (1997): Ein Ort Namens Kumait. Die Geschichte einer Beschneidung. Roman. Kairo.

- (2004): Die Reise nach Tell al-Lahm. Roman. Aus dem Arab. v. Imke AhlfWien. München.

- (2008): Jussifs Gesichter. Roman aus der Mekka-Bar. Aus dem Arab. v. Imke Ahlf-Wien. München.

- (2009): Reise in das Herz des Feindes. Ein Iraker in Israel. Roman. Aus dem Arab. v. Imke Ahlf-Wien. München.

- (2011): Engel des Südens. Roman. Aus dem Arab. v. Imke Ahlf-Wien. München.

- (2014): Bagdad Marlboro. Roman. Aus dem Arab. v. Hartmut Fähnrich. München.

- (2015): Bagdad. Erinnerungen an eine Weltstadt. Roman. Aus dem Arab. v. Hartmut Fähnrich. München.

\section{LITERATUR}

Ash-Shibibi, Jamil (o.J.): Identitätsproblem im zeitgenössischen irakischen Roman. Identitätssuche in einem versunkenen Land; online unter: http://www.ahewar.org/ [Stand: 1.4.2020].

Ḥaddad, Lutfi (2004): Ontologie der zeitgenössischen arabischen Exilliteratur. Beirut. Hessenland, Frank (2006): Literatur und Krieg. Treffen von irakischen Exil-SchriftstelIern in Berlin. In: deutschlandfunk.de, 26. Februar 2006; online unter: https://www. deutschlandfunk.de/literatur-und-krieg.691.de.html?dram:article_id=49445 [Stand: 1.4.2020]. 
Ibrahim, Abdullah (2009): Zum Exil und irakischer Exilliteratur. In: Al-Ra'i Zeitung v. 12. März 2009.

Löffler, Sigrid (2014): Erzählen vom unerzählbaren Inferno. Ein Iraker und ein US-Amerikaner kämpfen mit dem eigenen Schuldbewusstsein. In seinem großen Kriegsroman "Bagdad Marlboro" schaut Najem Wali auf ein tiefes Trauma. In: Salzburger Nachrichten v. 5. April 2014.

Thiab, Safa'a (2014): Die Verwandlung des irakischen Romans und die Wende. In: AlSabah Zeitung v. 14. Januar 2014.

Tuckermann, Anja (Hg.; 1989): In die Flucht geschlagen. Geschichten aus dem bundesdeutschen Asyl. Frankfurt a.M. 
